\title{
Knowledge, Attitude, and Practice of Nurses Toward Sexual Guidance on Patients After Total Hip Replacement: Preliminary Survey Results
}

\author{
Jiayu Zheng ${ }^{1}$, Cuiqing Liu ${ }^{2}$, Yanfei $\mathrm{Li}^{1,}$ * \\ ${ }^{1}$ Department of Spinal and Trauma, the First Affiliated Hospital of Jinan University, Guangzhou, China \\ ${ }^{2}$ Department of Nursing, the First Affiliated Hospital of Jinan University, Guangzhou, China \\ Email address: \\ 2212894241@qq.com (Jiayu Zheng),973517560@qq.com (Yanfei Li) \\ ${ }^{*}$ Corresponding author
}

To cite this article:

Jiayu Zheng, Cuiqing Liu, Yanfei Li. Knowledge, Attitude, and Practice of Nurses Toward Sexual Guidance on Patients After Total Hip Replacement: Preliminary Survey Results. American Journal of Nursing Science. Vol. 9, No. 3, 2020, pp. 181-185.

doi: 10.11648/j.ajns.20200903.29

Received: May 1, 2020; Accepted: May 21, 2020; Published: May 29, 2020

\begin{abstract}
To investigate the knowledge, attitude, and behavior of nurses toward sexual guidance on patients after THR (total hip replacement) surgery, thus providing data for departments to develop nursing policies for patients after THR. A Web-based survey was completed by 92 nurses from the First Affiliated Hospital of Jinan University. Viability analysis was conducted to evaluate the questionnaire's reliability. Data were collected and explored by descriptive analysis and analysis of variance to assess the knowledge, attitude, and behavior of nurses with different characteristics toward sexual guidance for patients after THR. The survey showed that the nurses with more working experience got a higher score on knowledge. And orthopedic specialist nurses score higher than non-specialist nurses. The difference was statistically significant $(P<0.05)$. The attitude and behavior scores were very low among the nurses of all groups with different characteristics, they did not reach a total score of $60 \%$, and there was no significant statistical difference between all groups $(P>0.05)$. In conclusion, the nurses with more working experience or specialize in orthopedic department have a good knowledge of sexual guidance on patients after THR. But nurses with different characteristics have a low score of attitude and behavior scores on of sexual guidance on patients after THR. The clinical department should improve training and emphasize the importance of sexual guidance on patients after THR.
\end{abstract}

Keywords: Knowledge, Attitude, Practice, Sexual Guidance, Total Hip Replacement

\section{Introduction}

THR is a surgery to replace the damaged hip joint with the artificial hip joint prostheses. It provides an effective and feasible treatment for severe hip joint lesions [1, 2]. Once the dislocation of the prosthesis occurs after THR, the joint should undergo external fixation for 6 weeks under anesthesia, Even worse, severe cases should undergo revision surgery, while the incidence of prostheses dislocation after revision can reach $28 \%$ $[3,4]$. Thus, dislocation of the prosthesis will not only endanger hip function but also increase medical expenses, lengthen hospital stay, occupy the limited medical resources [5]. Therefore, prevention of dislocation of the prosthesis after hip replacement is one of the focuses of orthopedics.
Nurses' inadequate instructions on exercise for patients after THR may lead to the occurrence of the hip prosthesis dislocation [5]. Hence, post-THR limb rehabilitation training has attracted the attention of medical administrations. As nurses are more conservative in sex, instructions about physical rehabilitation have been emphasized, while sexual guidance for patients with sexual needs after THR are neglected. The American nursing association has clearly stipulated that attention of sex education on patients is one of the legal aspects of nursing work [6]. Sexual guidance for patients after THR can not only effectively prevent hip dislocation and other implications, but also improve the patients' quality of life.

At present, the knowledge-attitude-practice model has been 
applied in the medical and nursing fields, showing its feasibility and effectiveness [7-9]. Nonetheless, there are few reports of nurses' knowledge, attitude, and behavior about sexual health education after THR [10]. This study aims to understand the current situation of knowledge, attitude, and behavior of orthopedic nurses toward sexual guidance after THR, thus providing a reference for the training, intervention, and management of nursing work after THR.

\section{Methods}

\subsection{Survey Samples}

The sample consist of 92 nurses form the First Affiliated Hospital of Jinan University were selected as the subjects of investigation. The inclusion criteria are as follows: (1) Registered nurse, (2) Nurses worked in orthopedics for more than 1 year, (3) Informed and agreed to participate in this study. The exclusion criteria are as follows: (1) Absence during the survey period, (2) During rotation or regulation period.

\subsection{Survey Design}

The questionnaire was prepared, the general information items include gender, age, working years, education, professional title, specialist nurse in orthopedics or not.

The questionnaire to assess the knowledge, attitude, and behavior of nurses toward sexual guidance after hip replacement was combined with expert opinion. 4 chief nurses, 4 chief physicians, and 2 deputy chief nurses were invited to evaluate validity, and finally determined as 24 items. 19 nurses from the First Affiliated Hospital of Jinan University were selected for pre-survey to assess the reliability. The survey subjects should be comprehensible correctly, and answer be optional. The knowledge subjects includes 8 items such as sexual positions that should be prevented during the sexual life of patients after THR, labor-saving sexual position, and sexual position that obese patients should adopt. Each question gets a full score of 4 points if it is correct. Total score is 32 points, and the higher the score, the better the nurse has knowledge of sexual education after THR. The attitude items includes 8 items such as the necessity and importance of sexual guidance for patients after THR, whether give up instructions by patients and their families' negative attitude or not, and whether the ward environment matters or not for guiding. "Disagree Strongly" to "agree Strongly" scores 1 to 4 points. The higher the score, the more positive the nurse was. The behavior items includes 8 items, such as the frequency of sexual life that patients should have, sexual guidance for patients after THR operation or not, the importance of publicity on the prevention of hip dislocation, and provide educational books for patients. "Not" to "always" were assigned 1 to 4 points.

\subsection{Survey Procedure}

This survey was completed through the WeChat network platform

(https://www.wjx.cn/m/70325680.aspx?from=singlemessage \&isappinstalled $=0$ ). The consent was obtained by the hospital. The head nurse of the orthopedics department explains the purpose and significance of the survey to the nurses who meet the inclusion criteria. After obtaining consent, the questionnaire link is issued through the WeChat platform, which is filled out voluntarily. The questionnaire can only be submitted after answering, and each IP address can only fill in for once. The questionnaires which showed obvious regularity and the obvious tendency were excluded. A total of 114 questionnaires were completed, of which 92 were valid, and the effective rate was $80.70 \%$.

\subsection{Statistical Analysis}

Data were analyzed by SPSS 23.0, and the score was described by mean \pm standard deviation. The score was tested by normal distribution and one-way ANOVA test was used to compare the knowledge, attitude, and behavior scores. Correlation of nurses' knowledge, attitude and behavior was analyzed by bivariate correlations analysis. $P<0.05$ was considered statistically significant.

\section{Results}

\subsection{General Information and Characteristics of Nurses}

Among the 92 nurses, there are 14 nurses aged $<25$ years old, accounting for $15.2 \%, 46$ people aged $25-35$, accounting for $50 \%$ of the total. There are 32 people over 35 years old, accounting for $34.8 \%$ of the total. Gender: there are 82 women, accounting for $96.74 \%, 3$ males accounting for $3.3 \%$. Education: there are 34 nurses underwent secondary schools, accounting for $37.0 \%, 34$ college students, accounting for $37 \%, 22$ undergraduates, accounting for $23.9 \%, 2$ persons with a master degree or above, accounting for $2.2 \%$. Title: there are 18 assistants, accounting for $19.6 \%, 40$ juniors, accounting for $43.5 \%, 22$ intermediate, accounting for $23.9 \%$ and 12 Sub-senior or above, accounting for $13 \%$. Years of participation in nursing work: $<5$ years, there are 37 people, accounting for $23.9 \%$, 30 people for 5-10 years, accounting for $32.6 \%$. $>40$ people for 10 years, accounting for $43.5 \%$. There are 39 orthopedic specialists, accounting for $42.4 \%$ and 53 non-orthopedic specialists, accounting for $57.6 \%$.

\subsection{Nurses' Knowledge, Attitude, and Behavior Scores of Patients After Hip Replacement}

The scores of nurses with different population characteristics are shown in Table 2. Among them, it is worth noting that the nurses who work longer in orthopedics got a higher knowledge score (the score of nurses worked $<5$ years is $18.19 \pm 2.65,5-10$ years is $20.37 \pm 2.18$, and over 10 years is $24.71 \pm 3.11$. The difference was statistically significant $(P$ $<0.05)$. The score of the orthopedic specialist nurse $(24.04 \pm$ 2.58) was higher than that of the non-orthopedic specialist nurse $(17.47 \pm 1.78)$, The difference was statistically significant $(P<0.05)$. There was no statistical difference between the attitude score and behavior score of nurses with different characteristics $(P>0.05)$. 


\subsection{Correlation Analysis of Nurses' Knowledge, Attitude and Behavior of Patients After Hip Replacement}

In this sample, the mean and standard deviation of knowledge score was $22.70 \pm 2.70$ (total score 32 ), attitude is $15.38 \pm 2.95$ (32 points), and behavior is $13.59 \pm 6.68$ (total score 32). Among them, the average score of attitude and behavior is less than $60 \%$ of the total score. The minimum, maximum, mean, standard deviation, and Pearson coefficient are shown in Table 2. There was no significant correlation between knowledge attitude and behavior scores in this study.

Table 1. Different demographic characteristics on sexual life guidance knowledge, attitude, and behavior score of patients after hip replacement.

\begin{tabular}{|c|c|c|c|c|c|c|c|c|c|c|}
\hline & $\mathbf{n}$ & Knowledge & $\boldsymbol{F}$ & $\boldsymbol{P}$ & Attitude & $\boldsymbol{F}$ & $\boldsymbol{P}$ & Behavior & $\boldsymbol{F}$ & $P$ \\
\hline \multicolumn{11}{|l|}{ Age (years) } \\
\hline$\leq 25$ & 14 & $20.43 \pm 2.44$ & \multirow{3}{*}{0.097} & \multirow{3}{*}{0.908} & $15.64 \pm 2.65$ & \multirow{3}{*}{0.290} & \multirow{3}{*}{0.749} & $15.86 \pm 8.58$ & \multirow{3}{*}{2.468} & \multirow{3}{*}{0.091} \\
\hline $25-35$ & 46 & $22.70 \pm 2.76$ & & & $15.52 \pm 2.78$ & & & $12.11 \pm 6.01$ & & \\
\hline$>35$ & 32 & $22.69 \pm 2.70$ & & & $15.06 \pm 3.35$ & & & $14.72 \pm 6.40$ & & \\
\hline \multicolumn{11}{|l|}{ Education } \\
\hline Secondary & 34 & $19.82 \pm 2.92$ & \multirow{4}{*}{0.053} & & $15.21 \pm 2.08$ & & & $14.15 \pm 6.37$ & & \\
\hline College & 34 & $22.62 \pm 2.72$ & & \multirow{3}{*}{0.984} & $15.64 \pm 2.95$ & \multirow{3}{*}{0.149} & \multirow{3}{*}{0.930} & $13.06 \pm 6.96$ & \multirow{3}{*}{0.164} & \multirow{3}{*}{0.921} \\
\hline Bachelor & 22 & $22.59 \pm 2.52$ & & & $15.27 \pm 3.50$ & & & $13.64 \pm 6.97$ & & \\
\hline Master or above & 2 & $22.140 \pm 1.41$ & & & $15.00 \pm 9.90$ & & & $12.50 \pm 9.19$ & & \\
\hline \multicolumn{11}{|l|}{ Professional title } \\
\hline Assistant & 18 & $19.33 \pm 2.54$ & & & $15.61 \pm 3.27$ & & & $15.39 \pm 7.82$ & & \\
\hline Junior & 40 & $19.45 \pm 2.97$ & \multirow[b]{2}{*}{0.629} & \multirow[b]{2}{*}{0.598} & $15.45 \pm 2.30$ & \multirow[b]{2}{*}{0.276} & \multirow[b]{2}{*}{0.842} & $12.30 \pm 6.64$ & \multirow[b]{2}{*}{1.136} & \multirow[b]{2}{*}{0.339} \\
\hline Intermediate & 22 & $22.32 \pm 2.53$ & & & $15.45 \pm 3.49$ & & & $13.64 \pm 5.98$ & & \\
\hline \multicolumn{11}{|l|}{ Service years } \\
\hline$\leq 5$ & 37 & $18.19 \pm 2.65$ & \multirow{3}{*}{4.516} & \multirow{3}{*}{0.003} & $15.19 \pm 3.99$ & \multirow{3}{*}{0.159} & \multirow{3}{*}{0.854} & $14.54 \pm 7.42$ & \multirow{3}{*}{1.744} & \multirow{3}{*}{0.181} \\
\hline $5-10$ & 27 & $20.37 \pm 2.18$ & & & $15.41 \pm 3.04$ & & & $11.59 \pm 5.58$ & & \\
\hline$>10$ & 28 & $24.71 \pm 3.11$ & & & $15.61 \pm 2.74$ & & & $14.25 \pm 6.45$ & & \\
\hline \multicolumn{11}{|c|}{ Specialize in orthpedics } \\
\hline Yes & 39 & $24.04 \pm 2.58$ & \multirow[t]{2}{*}{5.175} & \multirow[t]{2}{*}{0.000} & $15.36 \pm 3.67$ & \multirow[t]{2}{*}{0.004} & \multirow[t]{2}{*}{0.953} & $14.0 \pm 06.23$ & 0.256 & 0.614 \\
\hline No & 53 & $17.47 \pm 1.78$ & & & $15.40 \pm 2.32$ & & & $13.28 \pm 7.04$ & & \\
\hline
\end{tabular}

Table 2. The means, standard deviations, and Pearson's correlation coefficients ( $n=92)$.

\begin{tabular}{|c|c|c|c|c|c|c|c|}
\hline & $M$ & $S D$ & Minimum & Maximum & Knowledge & Attitude & Behavior \\
\hline Knowledge & 22.70 & 2.71 & 13 & 32 & 1 & & \\
\hline Attitude & 15.38 & 2.95 & 6 & 24 & 0.039 & 1 & \\
\hline Behavior & 13.59 & 6.68 & 2 & 32 & 0.209 & 0.110 & 1 \\
\hline
\end{tabular}

\section{Discussion}

THR is a surgery to replace the damaged hip joint with the artificial hip joint prostheses. As nurses are more conservative in sex, instructions about physical rehabilitation have been emphasized, while sexual guidance for patients with sexual needs after THR are neglected [11]. This study aims to observe the current situation of knowledge, attitude, and behavior of orthopedic nurses toward sexual guidance after THR, thus providing a reference for the training, intervention, and management of nursing work after THR.

The orthopedic nurses have a general knowledge of sexual life guidance for patients after total hip replacement. The total score of the knowledge in this study was $0-32$, and the score of the knowledge of 92 orthopedic nurses in this study was 22.70 \pm 2.71 . Among them, the knowledge score is related to the number of years of orthopedic nursing work, and the longer the time to participate in orthopedic nursing work, the higher the score. In this study, the average score of orthopedic specialist nurses $(24.04 \pm 2.58)$ was higher than that of non-specialist nurses $(17.47 \pm 1.78)$, and the difference was statistically significant. Therefore, nursing managers should provide orthopedic nurses with targeted sexual guidance for patients after THR to shorten the learning period thus avoid postoperative complications caused by inappropriate sexual behaviors. Strengthen the clinical application of orthopedic nurses' knowledge in this area, and emphasize the importance of correct sex life to prevent postoperative complications of THR.

The orthopedic nurse's attitude towards sexual guidance after THR was $15.38 \pm 2.95$ (total score of 32 points), and the behavior score was $13.59 \pm 6.68$ (total score of 55 points). Even worse, they did not reach a total score of $60 \%$. There were no significant differences in the scores of nurse groups of different characteristics. Sexual life is a normal physiological activity of human beings and an essential part of high-quality life. Whether the patient can take correct sexual behavior after THR is closely related to the correct guidance of the orthopedic nurses. However, because of the influence of 
traditional values, the attitude towards sexual behavior after THR needs to be further improved, and guidance is very inadequate, which leads to unnecessary complications. The American Nursing Association has clearly stipulated that attention to sex is a legal part of nursing work [6]. Sexual guidance after THR can not only effectively prevent the occurrence of hip dislocation, but also benefit the physical and mental recovery of patients and improve the quality of life of patients [12-15]. The preliminary findings of this study show that orthopedic nurses with different population characteristics have poor attitudes and behavior towards sexual behavior guidance for patients after THR. Therefore, nursing managers should emphasize the importance and necessity of sexual life guidance after THR, break the traditional barrier, and improve the nursing after THR.

The knowledge-attitude-behavior model illustrates the link between knowledge, attitude, and behavior that is knowledge is the foundation, belief is the motivation, and behavior change is the goal [15-19]. The results of this study showed that there is no significant correlation with their knowledge, attitudes, and behaviors regarding sexual guidance for patients after total hip replacement. This may be ascribed to the conflict of traditional value and the nurse think it is not important and necessary of sexual guidance after THR. While the nurses in this study are all from China, they may also be influenced by Chinese traditional culture, which makes nurses shy to give sexual guidance to patients with THR.

Therefore, in the future, the clinical depatments should correct the nurse's attitude towards THR postoperative sexual guidance by training, thus making nurses recognize and believe internally that THR postoperative sexual guidance can improve the quality of life of patients and reduce the occurrence of complications. It is also recommended that medical schools should add the courses related to sexual guidance after THR. Clinical administrators should provide theoretical knowledge, evaluation, and training for new nurses. Therefore, the management department should regularly carry out correspondent policies by continuous improvement of standard evaluation and promote the application of sexual life guidance for patients after THR, thereby improving patient prognosis and improving the quality of life of patients.

\section{Conclusion}

The nurses with more working experience or specialize in orthopedic department have a good knowledge of sexual guidance on patients after THR. But nurses with different characteristics have a low score of attitude and behavior scores on of sexual guidance on patients after THR. Taken together, the clinical department should improve training and emphasize the importance of sexual guidance on patients after THR.

\section{Conflicts of Interest Statement}

All the authors do not have any possible conflicts of interest.

\section{References}

[1] Learmonth I D, Young C, and Rorabeck C. The operation of the century: total hip replacement. Lancet (London, England) [J]. 2007, 370 (9597): 1508-1519.

[2] Dattani R. Femoral osteolysis following total hip replacement. Postgraduate medical journal [J]. 2007; 83 (979): 312-316.

[3] Dargel J, Oppermann J, Brüggemann G P, et al. Dislocation following total hip replacement. Deutsches Arzteblatt international [J]. 2014, 111 (51-52): 884-890.

[4] Lu Y, Xiao H, and Xue F. Causes of and treatment options for dislocation following total hip arthroplasty. Experimental and therapeutic medicine [J]. 2019; 18 (3): 1715-1722.

[5] Rowan F E, Benjamin B, Pietrak J R, et al. Prevention of Dislocation After Total Hip Arthroplasty. The Journal of arthroplasty [J]. 2018, 33 (5): 1316-1324.

[6] Steinke E E, Jaarsma T, Barnason S A, et al. Sexual counselling for individuals with cardiovascular disease and their partners: a consensus document from the American Heart Association and the ESC Council on Cardiovascular Nursing and Allied Professions (CCNAP). European heart journal [J]. 2013, 34 (41): 3217-3235.

[7] Bogale A A, Amhare A F, Chang J, et al. Knowledge, attitude, and practice of self-medication with antibiotics among community residents in Addis Ababa, Ethiopia. Expert review of anti-infective therapy [J]. 2019, 17 (6): 459-466.

[8] Nisa Z U, Zafar A, and Sher F. Assessment of knowledge, attitude and practice of adverse drug reaction reporting among healthcare professionals in secondary and tertiary hospitals in the capital of Pakistan. Saudi pharmaceutical journal: SPJ: the official publication of the Saudi Pharmaceutical Society [J]. 2018, 26 (4): 453-461.

[9] Nasiri A, Balouchi A, Rezaie-Keikhaie K, et al. Knowledge, attitude, practice, and clinical recommendation toward infection control and prevention standards among nurses: A systematic review. American journal of infection control [J]. 2019, 47 (7): 827-833.

[10] Kotronoulas G, Papadopoulou C, and Patiraki E. Nurses' knowledge, attitudes, and practices regarding provision of sexual health care in patients with cancer: critical review of the evidence. Supportive care in cancer: official journal of the Multinational Association of Supportive Care in Cancer [J]. 2009; 17 (5): 479-501.

[11] Pascual A, Wighman A, Littooij E C, et al. Sexuality as part of rehabilitation? A qualitative study on the perceptions of rehabilitation nurses on discussing patient sexuality during clinical rehabilitation. Disability and rehabilitation [J]. 2019: $1-8$.

[12] Meiri R, Rosenbaum T Y, and Kalichman L. Sexual Function before and after Total Hip Replacement: Narrative Review. Sexual medicine [J]. 2014, 2 (4): 159-167.

[13] Lu Y, Wu Z, Tang X, et al. Effect of articular capsule repair on postoperative dislocation after primary total hip replacement by the anterolateral approach. The Journal of international medical research [J]. 2019; 47 (10): 4787-4797. 
[14] Sexuality and reproductive health in adults with spinal cord injury: a clinical practice guideline for health-care professionals. The journal of spinal cord medicine [J]. 2010; 33 (3): 281-336.

[15] Ghani N A, Shohaimi S, Hee A K-W, et al. Comparison of Knowledge, Attitude, and Practice among Communities Living in Hotspot and Non-Hotspot Areas of Dengue in Selangor, Malaysia. Tropical medicine and infectious disease [J]. 2019, 4 (1).

[16] Bettinghaus E P. Health promotion and the knowledge-attitude-behavior continuum. Preventive medicine [J]. 1986, 15 (5): 475-491.

[17] Di Simone E, Giannetta N, Auddino F, et al. Medication Errors in the Emergency Department: Knowledge, Attitude, Behavior, and Training Needs of Nurses. Indian journal of critical care medicine: peer-reviewed, official publication of Indian Society of Critical Care Medicine [J]. 2018, 22 (5): 346-352.

[18] Liu L, Liu Y-P, Wang J, et al. Use of a knowledge-attitude-behaviour education programme for Chinese adults undergoing maintenance haemodialysis: Randomized controlled trial. The Journal of international medical research [J]. 2016; 44 (3): 557-568.

[19] Xu W, Sun G, Lin Z, et al. Knowledge, attitude, and behavior in patients with atrial fibrillation undergoing radiofrequency catheter ablation. Journal of interventional cardiac electrophysiology: an international journal of arrhythmias and pacing [J]. 2010; 28 (3): 199-207. 